\title{
Nonparametric stochastic modeling of linear systems with prescribed variance of several natural frequencies
}

\author{
M.P. Mignolet \\ Arizona State University, Tempe, AZ 85287-6106, USA \\ C. Soize \\ Université de Marne-la-Vallée, 77454 Marne-la-Vallée, France
}

\begin{abstract}
A complete probabilistic model of random positive definite matrices is developed that incorporates constraints on the standard deviations of a set of its eigenvalues. The model is in particular applicable to the representation of the mass and stiffness matrices of random dynamic systems of which certain natural frequencies are observed. The model development is based on the maximization of the entropy under a set of constraints representing the prescribed eigenvalue standard deviations, the mean matrix being given, and the existence of the mean Frobenius norm of the inverse of the random matrix. The efficient simulation of samples of random matrices according to the proposed model is discussed in detail. Finally, examples of application validate the above concepts and demonstrate the usefulness of the proposed model.
\end{abstract}

Keywords: structural dynamics, random systems, random matrices, maximum entropy, probabilistic model

\section{INTRODUCTION}

The stochastic modeling and simulation of random multi degree of freedom systems has often in the past been accomplished by postulating joint probability density functions of the components of their mass, stiffness, and/or damping matrices or, equivalently, of their corresponding natural frequencies, damping ratios, and mode shapes (e.g., Ghanem and Spanos, 1991; Kleiber et al., 1992; Rivas-Guerra and Mignolet, 2004; Schueller, 1997). However, a different approach has recently been proposed (Soize, 2000; 2001) and validated (see Soize, 2005, for a review) in which the probabilistic model of the mass, stiffness, and/or damping matrices is not assumed but rather determined to maximize the entropy under the constraints (i) that these matrices are positive definite, (ii) that their mean values are prescribed, and (iii) of an overall measure of variation (variance of the norms of the matrices prescribed). This approach, which has been named nonparametric since no parameter value is selected by the user, is applicable to a broad range of applications in which little is known about the variability of the system. This approach will be referred to as unconstrained in the remainder of this paper.

There are many other situations as well in which more information is known about the variations of the system. For example, some routine testing may be done on at least part of the system (e.g., blades in turbomachinery applications) or an extensive testing may be performed on a small sample to better understand an observed problem or avoid one from occurring. Such testing often focuses on the natural frequencies and thus yields estimates of their means and variances. Since the mean natural frequencies are likely to be close to the values obtained for the design configurations, it is typically the variances which provide the best information about the system randomness.

In this light, the goal of the present investigation is to extend the formulation of the nonparametric approach to allow for additional constraints on the standard deviations of some of the eigenvalues of the mass, damping, and/or stiffness matrices. This novel approach will be referred to as constrained in the remainder of the paper.

Note that the expected domain of application of these concepts is the low frequency range in which the natural frequencies distinguishable and observable.

\section{ENTROPY MAXIMIZATION AND CONSTRAINTS}

It is desired here to simulate realizations of symmetric positive definite random matrices, e.g. the mass and/ or the stiffness matrix of a linear dynamic system, the properties of which, i.e., eigenvalues, eigenvectors, components, etc., are all random. This requirement necessitates the specification of the joint probability density function of all elements of the matrix. In most practical problems, however, this information is not available - only some moments and/or marginal probability density functions are likely to be available. In the absence of the exact distribution, it is then appropriate to ask what are the desirable features of this distribution. In this context, note that the design 
of structural systems is often robust, i.e., that small perturbations in their geometrical and material properties do not alter significantly the probability of failure/fatigue life of the system considered (see Rivas-Guerra and Mignolet, 2004 for a notable counterexample in turbomachinery). It is thus desirable to dispose of a probabilistic model which places particular emphasis on "larger" deviations from the design conditions. Equivalently, this model should have a large value of the entropy.

Consistently with the above discussion, a probabilistic model of uncertain $n x n$ matrices $A$ has been formulated (Soize, 2000; 2001) to maximize the value of the entropy $S$

$$
S=-\int_{\Omega} p_{\underline{\underline{A}}}(\underline{\underline{a}}) \ln p_{\underline{\underline{A}}}(\underline{\underline{a}}) d \underline{\underline{a}}
$$

given the following physical constraints:

$$
\begin{aligned}
& \int_{\Omega} p_{\underline{\underline{A}}}(\underline{\underline{a}}) d \underline{\underline{a}}=1 \\
& E[\underline{\underline{A}}]=\int_{\Omega} \underline{\underline{a}} p_{\underline{\underline{A}}}(\underline{\underline{a}}) d \underline{\underline{a}}=\underline{\underline{A}}
\end{aligned}
$$

and

$$
\int_{\Omega} \ln [\operatorname{det}(a)] p_{\underline{\underline{A}}}(a) d \underline{\underline{a}}=\nu \text { finite }
$$

where $E[$.$] denotes the operation of mathematical$ expectation, $p_{\underline{A}}(\underline{\underline{a}})$ is the joint probability density function of the elements of $\underline{\underline{A}}$, and $\operatorname{det}(\underline{\underline{A}})$ is its determinant.

The first two of the above constraints correspond to the normalization of the total probability to 1 (Eq. (2)) and the specification of the mean matrix (Eq. (3)). The third one, Eq. (4), implies the existence of the mean squared Frobenius norm of the inverse matrix $A^{-1}$ (see Soize $(2000,2001)$ for discussion). To apply this approach to the simulation of random mass, stiffness, and damping matrices of dynamical systems, it is further required to ensure both the symmetry and positive definiteness of every realized matrix $\underline{A}$. This is achieved by introducing the Cholesky decomposition of $\underline{\underline{A}}$ i.e.,

$$
\stackrel{A}{=} \underline{\underline{\tilde{L}}} \tilde{L}^{T}
$$

where $\tilde{L}$ is an lower triangular matrix with nonnegative diagonal elements and ${ }^{T}$ denotes the operation of matrix transposition. The domain of support
$\Omega$ of the obtained probability density function is then such that the elements $\tilde{L}_{i j}$ belong to $(-\infty,+\infty)$ for $i \neq j$ and $[0,+\infty]$ for $i=j$ i.e.,

$$
\begin{aligned}
\Omega= & \left\{\underline{\underline{a}}=\underline{\underline{L}} \underline{\underline{L}}^{T} ; \tilde{L}_{i j}, i, j=1, \ldots, n:\right. \\
& {\left.\left[\tilde{L}_{i j} \in(-\infty,+\infty), i>j\right] \cap\left[\tilde{L}_{i i} \in(0,+\infty)\right]\right\} }
\end{aligned}
$$

Not only have the theoretical aspects of this formulation been discussed extensively but it also been broadly validated and applied (see Soize (2005) for a review). Note in the above approach that the level of uncertainty is controlled by the single parameter $v$ so that only a broad knowledge of the matrix uncertainty needs to be known. In some situations however, e.g., when considering insertable turbomachinery blades, tests may have been performed that provide more information on the system variability. In the context of structural dynamics, such tests will often focus on the natural frequencies of the system and would result in estimates of the variance of the first few natural frequencies. In such circumstances, it is highly desirable to dispose of a probabilistic model of the corresponding mass and stiffness matrices that accurately accounts for this information. If only one natural frequency is observed, its variance as estimated by the tests can serve for the determination of the parameter $v$, Eq. (4), corresponding to the mass and/or stiffness matrix model. However, to account for two or more variances, it is necessary to extend the formulation of Eqs. (1)-(4) by introducing additional constraints that can reflect the knowledge on the natural frequencies. To address this extension, consider the generalized eigenvalue problems

$$
\underline{\underline{A}} \underline{\varphi}_{i}=\lambda_{i} \underline{\underline{B}} \underline{\underline{\varphi}}_{i} \text { and } \underline{\underline{A}} \underline{\underline{\phi}}=\tilde{\lambda}_{i} \underline{\underline{B}} \underline{\phi}_{i}
$$

where $\underline{B}$ is a deterministic symmetric, positive definite matrix. In the ensuing discussions, it will be assumed that the eigenvectors $\underline{\varphi}_{i}$ and $\underline{\phi}_{i}$ are normalized with respect to $\underline{\underline{B}}$ so that

$$
\underline{\varphi}_{i}^{T} \underline{B}_{i}=1 \quad \underline{\varphi}_{i}^{T} \underline{A}_{i}=\underline{\varphi}_{i}
$$

and

$$
\underline{\phi}_{i}^{T} \underline{\underline{B}} \underline{\phi}_{i}=1 \quad \underline{\phi}_{i}^{T} \underline{\underline{A}} \underline{\phi}_{i}=\tilde{\lambda}_{i} .
$$

Constraining the variance of the eigenvalues $\lambda_{i}$ directly is unfortunately extremely challenging because of the lack of an exact expression for the natural frequencies of the random matrix $\underline{\underline{A}}$. Accordingly, an 
indirect approach will be selected here which relies on simple constraints that are akin to the second order moments of the eigenvalues. More specifically, it will be assumed that

$$
E\left[\left(\underline{\phi}_{i}^{T} \underline{\underline{A}} \underline{\phi_{i}}\right)^{2}\right]=\int_{\Omega}\left(\underline{\phi}_{i}^{T} \underline{\underline{a}} \underline{\phi}_{i}\right)^{2} p_{\underline{\underline{A}}}(\underline{\underline{a}}) d \underline{\underline{a}}=s_{i}^{2} \tilde{\lambda}_{i}^{2}
$$

where $s_{i}, i \in I \subseteq[1, n]$, are $m$ known positive constants and $\underline{\phi}_{i}$ are the eigenvectors of the mean matrix $\underline{\underline{A}}$ corresponding to the $m$ eigenvalues of which the variance is known. For example, if the variances of the three lowest eigenvalues of $A$ have been estimated, then $\phi_{i}$ will similarly be the eigenvectors of the mean matrix $\underline{\underline{A}}$ corresponding to its three lowest eigenvalues.

Several comments can made in regards to the constraints of Eq. (10). First, these conditions involve second order moments, not variances, but this switch is appropriate because the mean values of $\left(\phi_{i}^{T} \underline{\underline{A}} \phi_{i}\right)$ are already prescribed by Eq. (3). Next, as discussed above, these conditions do not generally relate exactly to the natural frequencies of the random matrix $A$, but they do so when its eigenvectors are the same as those of its mean $\overline{\underline{A}}$. Finally, it should be noted that the specification of the constraints of Eq. (10) provides freedom in the probabilistic model of the random matrix $A$ which can be used to match the known variances of the natural frequencies. This last issue will be discussed in more details further.

\section{PROBABILISTIC MODEL DERIVATION}

Following the discussions of the previous sections, the proposed probabilistic model $p_{\underline{A}}(\underline{a})$ maximizes the entropy, $S$ of Eq. (1), under ${ }^{n}$ the constraints of Eqs. (2)-(4) and (10) as well as the symmetry and positive definiteness requirement of Eq. (5). Using Lagrange multipliers, $\mu_{0}, \mu \lambda-1$, and $\tilde{\tau}_{i}$ the constrained maximization of Eq. (1) is reduced to the unconstrained maximization of

$$
\begin{aligned}
S^{*}= & \left.S-\mu_{0} \int_{\Omega} p_{\underline{\underline{A}}}(\underline{\underline{a}}) d \underline{\underline{a}}-\operatorname{tr}\left\{\tilde{\tilde{\mu}}_{\underline{\underline{\mu}}}^{T} \int_{\Omega} \underline{\underline{a}} \underline{\underline{\underline{A}}} \underline{\underline{\underline{a}}} \underline{\underline{a}}\right) d \underline{\underline{a}}\right\} \\
& \left.+(\lambda-1) \int_{\Omega} \ln [\operatorname{det}(\underline{\underline{a}})] \underline{\underline{p_{A}}} \underline{\underline{a}}\right) d \underline{\underline{a}} \\
& \left.\left.-\sum_{i \in I} \tilde{\tau}_{i} \int_{\Omega}\left(\underline{\phi}_{i}^{T} \underline{\underline{a}} \underline{\underline{\phi}}\right)^{2}\right)^{2} \underline{\underline{p}}_{\underline{\underline{A}}} \underline{\underline{a}}\right) d \underline{\underline{a}}
\end{aligned}
$$

Proceeding next by calculus of variations, it is shown that

$$
p_{\underline{A}}(\underline{\underline{a}})=\tilde{C}[\operatorname{det}(\underline{\underline{a}})]^{\lambda-1} \exp \left[-\operatorname{tr}(\underline{\underline{\mu}} \underline{\underline{\underline{\mu}}} \underline{\underline{a}})-\sum_{i \in I} \tilde{\tau}_{i}\left(\underline{\phi}_{i}^{T} \underline{\underline{a}} \underline{\underline{\phi}} \underline{ }^{2}\right)^{2}\right]
$$

where $\widetilde{C}$ is the appropriate normalization constant to satisfy the normalization condition, Eq. (2). It should be noted from Eq. (12) that this probabilistic model is independent of a rotation/change of coordinate system as the vectors $\phi_{i}$ are fixed in space.

Before addressing the evaluation of the Lagrange multipliers, it is desired to simplify Eq. (12) and to address the positive definiteness requirement. In regards to simplifications, introduce first the matrix $\underline{\underline{L}}$ such that

$$
\underline{\underline{A}}=\underline{\underline{L}} \underline{\underline{L}}^{T} \quad \text { and } \quad \underline{\underline{L}}^{T} \underline{\underline{\Phi}}=\left[\begin{array}{l}
\tilde{\tilde{\Lambda}}^{1 / 2} \\
\underline{\underline{\underline{0}}}
\end{array}\right]
$$

where $\Phi$ denotes the $n \times m$ matrix formed by the $m$ eigenvectors $\phi, i \in I$, and $\tilde{\Lambda}$ is the corresponding diagonal matrix of eigenvalues. Note that the condition of Eq. (13b) is introduced to simplify the constraints of Eq. (10) as will be shown below. From Eqs. (6)-(9), it can be proved that $\bar{L}$ can be expressed in the partitioned form

$$
\underline{\underline{L}}=\left[\underline{\underline{A}} \underline{\underline{\Phi}} \underline{\underline{\Lambda}}^{-1 / 2} \underline{\underline{D}}\right]
$$

where the $n \times(n-m)$ matrix $\underline{D}$ is any decomposition, e.g. Cholesky, of

$\underline{\underline{D}} \underline{\underline{D}}^{T}=\underline{\bar{A}}-\underline{\bar{A}} \underline{\underline{\Phi}} \underline{\underline{\Lambda}}^{-1} \underline{\underline{\Phi}}{ }^{T} \underline{\underline{A}}$.

Pre- and postmultiplying Eq. (15) by $\underline{\Phi}^{T}$ and $\Phi$, respectively, it is found that $\underline{\underline{D}}^{T} \underline{\underline{\Phi}}=\underline{\underline{0}}$ as required in Eq. (13b).

Next, express the random matrix $\underline{\underline{A}}$ as

$\underline{\underline{A}}=\underline{\underline{L}} G \underline{\underline{\underline{L}}}$.

Proceeding with this change of random variables, it is found that the probability density function of the elements of $G$ is

$$
p_{\underline{\underline{G}}}(\underline{\underline{g}})=\bar{C}[\operatorname{det}(\underline{\underline{g}})]^{\lambda-1} \exp \left[-\operatorname{tr}(\underline{\underline{\mu}} \underline{\underline{g}})-\sum_{i=1}^{m} \tau_{i} g_{i i}^{2}\right]
$$

where $\bar{C}$ is a new normalization constant, $\underline{\underline{\mu}}=\underline{\underline{\underline{L}}} \underline{\underline{\mu}} \underline{\underline{\underline{L}}}$ and $\tilde{\tau}_{i}=\tilde{\tau}_{i} \tilde{\lambda}_{i}^{2}$.

To guarantee the symmetry and positive definiteness of $G$, and thus of $A$ the model of Eq. (17) is reformulated in terms of the elements of the lower triangular matrix $\underline{\underline{H}}$ such that

$$
\underline{\underline{G}}=\underline{\underline{H}} \underline{\underline{H}}^{T}
$$


As demonstrated in Soize (2000), the Jacobian of the transformation and the resulting distribution are

$$
\begin{aligned}
& J=2^{n} \prod_{l=1}^{n} h_{l l}^{n-l+1} \\
& p_{\underline{\underline{H}}}(\underline{\underline{h}})= C\left\{\prod_{l=1}^{n} h_{l l}^{n-l+2 \lambda-1}\right\} \\
& \times \exp \left[-\operatorname{tr}\left(\underline{\underline{h}}^{T} \underline{\mu}^{T} \underline{\underline{h}}\right)-\sum_{i=1}^{m} \tau_{i}\left(\sum_{l=1}^{i} h_{i l}^{2}\right)^{2}\right]
\end{aligned}
$$

where $C$ is the appropriate normalization constant over the domain

$$
\begin{aligned}
\bar{\Omega}= & \left\{h_{i j}, i, j=1, \ldots, n:\left[h_{i j} \in(-\infty, \infty), i>j\right]\right. \\
& \left.\cap\left[h_{i j} \in+[0,+\infty)\right]\right\} .
\end{aligned}
$$

To evaluate the Lagrange multipliers $\underline{\mu}$ and $\tau_{i}$, it is necessary to first express the constraints of Eqs. (3) and (10) in terms of the elements of the random matrix $\underline{\underline{H}}$. Combining Eqs. (3), (16), and (18), it is found that

$$
E[\underline{\underline{G}}]=\underline{=}
$$

where $I_{=n}$ denotes the $n \times n$ identity matrix. The simplicity of this condition implies an equally simple form of the matrix $\mu$ Specifically, it will be shown that this matrix is diagonal. Under this assumption, Eq. (20) reduces to

$$
\begin{aligned}
& p_{\underline{\underline{H}}}(h)=\prod_{i=1}^{m}\left\{C_{i} h_{i i}^{p(i)} \exp \left[-\mu_{i i}\left(\sum_{l=1}^{i} h_{i l}^{2}\right)-\tau_{i}\left(\sum_{l=1}^{i} h_{i l}^{2}\right)^{2}\right]\right\} \\
& \times \prod_{i=m+1}^{n}\left\{C_{i} h_{i i}^{p(i)} \exp \left[-\mu_{i i} h_{i i}^{2}\right]\right\} \times \prod_{i=m+1}^{n} \prod_{l=1}^{i-1}\left\{C_{i l} \exp \left[-\mu_{i i} h_{i l}^{2}\right]\right\}
\end{aligned}
$$

where $C_{i}, i=1, \ldots, n$, and $C_{i l}, i=m+1, \ldots, n ; l=1, \ldots$, $i-1$, are appropriate normalization constants and

$p(i)=n-i+2 \lambda-1$.

It is concluded from Eq. (22) that:

(i) the elements $h_{i i}, i>l$ and $i>m$ are all indepen dent of each "other and independent of the other elements $h_{i l}$. Further, they are normally distributed with mean 0 and standard deviation $\sigma_{i l}=\left(2 \mu_{i i}\right)^{-1 / 2}$.

(ii) the elements $h_{i i}, i>m$, are all independent of each other and independent of the other elements $h_{i l}$. Further, they are distributed according to

$$
p_{H_{i i}}\left(h_{i i}\right)=C_{i} h_{i i}^{p(i)} \exp \left|-\mu_{i i} h_{i i}^{2}\right|, h_{i i} \geq 0
$$

where $C_{i}=2 \mu_{i i}^{[p(i)+1] / 2} / \Gamma((p(i)+1) / 2)$

and $\Gamma($.) denotes the Gamma function.

(iii) the elements, $h_{i l}, l=1, \ldots, i$ for a given $i \in[1, m]$ are dependent on each other but independent of the other elements $h_{i l}$. Their joint distribution is

$$
p_{H_{i l}}\left(h_{i l}\right)=C_{i} h_{i i}^{p(i)} \exp \left[-\mu_{i i}\left(\sum_{l=1}^{i} h_{i l}^{2}\right)-\tau_{i}\left(\sum_{l=1}^{i} h_{i l}^{2}\right)^{2}\right]
$$

over the domain

$$
\begin{aligned}
& \bar{\Omega}_{i}=\left\{h_{i l}, l=1, \ldots, i:\left[h_{i l} \in(-\infty,+\infty), i>l\right]\right. \\
& \left.\cap\left[h_{i i} \in[0,+\infty)\right]\right\} .
\end{aligned}
$$

From the first observation, (i), it is concluded that $E\left[G_{i l}\right]=0$ for $i>l$ and $i>m$. Further, the observation (iii) and the symmetry of the distribution of Eq. (26) with respect to the origin imply similarly that $E\left[G_{i l}\right]=0$ for $i>l$ and $i \leq m$. It then remains to satisfy the diagonal terms of the mean condition, Eq. (21). For $i>m$,

$$
E\left[G_{i i}\right]=1=\sum_{l=1}^{i} E\left[H_{i l}^{2}\right]=(i-1) \frac{1}{2 \mu_{i i}}+E\left[H_{i i}^{2}\right]
$$

where $E\left|H_{i i}^{2}\right|$ is obtained by integration of Eq. (26) as $(p(i)+1) / 2 \mu_{i i}$. Combining this result and Eqs. (25) and (27), it is found that

$\mu_{i i}=(n+2 \lambda-1) / 2$ for $i>m$.

At this point, it only remains to determine the Lagrange multipliers $\mu_{i i}$ and $\tau_{i}$ for $i \in[1, m]$. This step is achieved by enforcing the conditions

$E\left[G_{i i}\right]=1$

and Eq. (11) or $E\left[G_{i i}^{2}\right]=s_{i}^{2}$

for $i \leq m$ To this end, it is useful to proceed with the following change of variables that highlights the random element $G_{i i}$

$$
\begin{aligned}
& G_{i i}=\sum_{l=1}^{i} H_{i l}^{2}, \quad H_{i i}=\sqrt{G_{i i}} \cos \Theta_{i 1} \\
& H_{i(i-1)}=\sqrt{G_{i i}} \sin \Theta_{i 1} \cos \Theta_{i 2} \\
& H_{i(i-2)}=\sqrt{G_{i i}} \sin \Theta_{i 1} \sin \Theta_{i 2} \cos \Theta_{i 3} \\
& H_{i 1}=\sqrt{G_{i i}} \sin \Theta_{i 1} \sin \Theta_{i 2} \ldots \sin \Theta_{i(i-1)}
\end{aligned}
$$

where $\Theta_{i 1} \in[0, \pi / 2], \Theta_{i l} \in[0, \pi], l=2, \ldots, i-2$, and $\Theta_{i(i-1)} \in[0,2 \pi)$. The Jacobian of this transformation can be found (e.g. see Soize, 2000) to be 


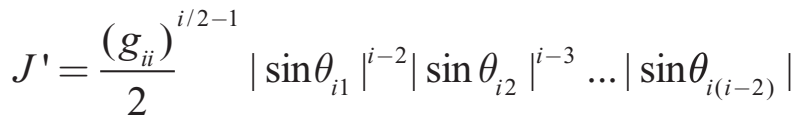

Then, the joint probability density function of $G_{i i}$ and $\Theta_{i l}, l=1, \ldots, i-1$, is

$$
\begin{aligned}
& p_{G_{i i} \Theta_{i l}}\left(g_{i i}, \theta_{i l}\right)=\frac{1}{2} C_{i} g_{i i}^{(n+2 \lambda-3) / 2} \exp \left[-\mu_{i i} g_{i i}-\tau_{i} g_{i i}^{2}\right] \\
& \quad \times\left|\sin \theta_{i 1}\right|^{i-2}\left[\cos \theta_{i 1}\right]^{p(i)} \times\left|\sin \theta_{i 2}\right|^{i-3} \ldots\left|\sin \theta_{i(i-2)}\right| .
\end{aligned}
$$

The above expression demonstrates that the random variables $G_{i i}$ and $\Theta_{i l}, l=1, \ldots, i-1$, are all independent of each other and that the joint probability density function of the angles $, \Theta_{i l}, l=1, \ldots, i-1$, does not depend on the values of $\tau_{i}$. This important observation will be used in the next section. Further, the marginal distribution of $G_{i i}$ is

$$
p_{G_{i i}}\left(g_{i i}\right)=\bar{C}_{i} g_{i i}^{(n+2 \lambda-3) / 2} \exp \left[-\mu_{i i} g_{i i}-\tau_{i} g_{i i}^{2}\right]
$$

where $g_{i i} \geq 0$ For each value of $i$, the two parameters $\mu_{i i}$ and $\tau_{i}$ are then finally solved from the constraints given by Eqs. (29) and (30).

\section{SIMULATION OF RANDOM MATRICES}

The simulation of random matrices $A$ according to the model derived above is achieved by first generating random matrices $H$ according to the joint distributions of Eqs. (22), (24), (26), (37) and (38). Once a sample of $H$ has been generated, the corresponding matrix $G \overline{\overline{\text { is }}}$ determined from Eq. (18) and, finally, a $\overline{\bar{r}}$ alization of $A$ is obtained from Eq. (16). Thus, the simulation effort reduces to the generation of appropriate samples of $H$. From the observations drawn in the previous section, it is concluded that there are, besides the simulation of the Gaussian variates $H_{i l}, i>m$ and $i>l$, three particular issues. These are: (i) the generation of the diagonal elements $H_{i i}, i>m$ according to the distribution of Eq. (24), (ii) the simulation of the ratios $H_{i l} / G_{i i}, i \leq m$, described by the angular variables in Eqs (32)-(37), and (iii) the generation of samples of $G_{i i}, i \leq m$, according to Eqs. (38). These three issues are addressed below.

\subsection{Simulation of $H_{i i}, i>m$}

The generation of samples of $H_{i i}$ is simplified by considering the variable $Y_{i i}=\mu_{i i} H^{2_{i i}}$. Proceeding with the change of variables, it is found that the probability density function of $Y_{i i}$ is

$p_{Y_{i i}}\left(y_{i i}\right)=\frac{y_{i i}^{(p(i)-1) / 2}}{\Gamma[(p(i)+1) / 2]} \exp \left[-y_{i i}\right], \quad y_{i i} \geq 0$.
Accordingly, it is found that $Y_{i i}$ is a Gamma distributed random variable for which efficient simulation algorithms exist, e.g. see Devroye (1986). Once a sample of $Y_{i i}$ has been simulated according to the Gamma distribution, the corresponding value of $H_{i i}, i>m$, is found as

$$
H_{i i}=\sqrt{Y_{i i} / \mu_{i i}}
$$

where $\mu_{i i}$ is given by Eq. (28).

\subsection{Simulation of $H_{i l} / \sqrt{G_{i i}}, i \leq m, l=1, \ldots, i$}

A first approach for the simulation of the random variables $H_{i l} / \sqrt{G}_{i i}, l=1, \ldots, i-1$, and $i \leq m$, is to proceed from the generalized spherical coordinates transformation of Eqs. (32)-(35) and to generate independent angles $\Theta_{i l}, l=1, \ldots, i-1$, according to the distributions

$p_{\Theta_{i 1}}\left(\theta_{i 1}\right)=\tilde{C}_{i 1}\left|\sin \theta_{i 1}\right|^{i-2}\left[\cos \theta_{i 1}\right]^{p(i)}$

and $p_{\Theta_{i l}}\left(\theta_{i l}\right)=\tilde{C}_{i l}\left|\sin \theta_{i l}\right|^{i-l-1}$

with, $\theta_{i 1} \in[0, \pi / 2], \quad \theta_{i l} \in[0, \pi]$ for $l=2, \ldots, i=2$ and $\theta_{i(i-1)} \in[0,2 \pi]$ and where the coefficients $\tilde{C}_{i l}$ are appropriate normalization constants.

Note however that the above distributions are non standard and thus a different, easier approach was selected here. Specifically, it was observed in the previous section that the probability density function of Eqs. (41) and (42) do not depend on $\tau_{i}$ and thus they would be the same for $\tau_{i}=0$. In this case however, the simulation of the random variables $H_{i l}$ is completely similar to the case $i>m$, i.e., the random variables, $H_{i l}, l \neq i$ are Gaussian variates with mean zero and standard deviation $1 / \sqrt{2 \mu_{i i}}$ while $H_{i l}=\sqrt{Y_{i i} / \mu_{i i}}$ where $Y_{i i}$ is a Gamma random variable. Note further that the appropriate value of $\mu_{i i}$ to be used is the one given by Eq. (28) to insure the consistency with $p(i)$ of Eq. (23).

In view of these comments, the simulation of the terms $H_{i l} / \sqrt{G_{i i}}, \quad l=1, \ldots, i-1$, and $i \leq m$, can efficiently be accomplished as follows:

(a) generate $\tilde{H}_{i i}$ as

$\tilde{H}_{i i}=\sqrt{\tilde{Y}_{i i} / \mu_{i i}}$

where $\mu_{i i}$ is given by Eq. (28) and $Y_{i i}$ is a Gamma distributed random variable of probability density function given by Eq. (39). 
(b) generate $\tilde{H}_{i l}, l=1, \ldots, i-1$, as zero mean Gaussian random variables with standard deviation $1 / \sqrt{2 \mu_{i i}}$.

(c) form $\tilde{G}_{i i}=\sum_{l=1}^{i} \tilde{H}_{i l}^{2}$ and the desired ratios

$$
H_{i l} / \sqrt{G_{i i}}=\tilde{H}_{i l} / \sqrt{\tilde{G}_{i i}} \quad l=1, \ldots, i .
$$

\subsection{Simulation of $G_{i i}, i \leq m$}

The last step in the simulation of the random matrix $\underline{\underline{H}}$ is the generation of the diagonal elements $G_{i i}$ according to their probability density function of Eq. (38). This distribution is non standard and thus a dedicated simulation algorithm by rejection from the Student's $t$ distribution with 3 degrees-of-freedom was devised (see Devroye (1986) for background). Such an algorithm requires that there exists a constant $c_{\theta}$ such that

$$
p_{G_{i i}}\left(g_{i i}\right) \leq c_{\theta} p_{Z_{\theta}}\left(g_{i i}\right) \text { for all } g_{i i} \geq 0
$$

where $p G_{i i}\left(g_{i i}\right)$ is defined by Eq. (38) and $p Z_{\theta}\left(g_{i i}\right)$ is the probability density function of $Z_{\theta}=y_{0}+\theta Z$ in which $Z$ is a random variable having the Student's $t$ distribution with 3 degrees-of-freedom. i.e., the probability density function of $Z_{\theta}$ is

$p_{Z_{\theta}}\left(g_{i i}\right)=\frac{1}{2 \sqrt{2} \theta}\left(1+\frac{\left(g_{i i}-y_{0}\right)^{2}}{2 \theta^{2}}\right)^{-3 / 2}$

for $g_{i i} \in(+\infty,-\infty)$. If values of $c_{\theta}, \theta$ and $y_{0}$ can be found for which Eq. (45) holds, then random deviates $G_{i i}$ can be generated as

$$
G_{i i}=Z_{\theta} \text { provided } c_{\theta} p_{Z_{\theta}}\left(Z_{\theta}\right) \bar{U} \leq p_{G_{i i}}\left(Z_{\theta}\right)
$$

in which $\bar{U}$ denotes a random variable uniform in the interval $[0,1]$ and is independent of $Z_{\theta}$. When the inequality in Eq. (47) is not satisfied, no sample $G_{i i}$ is generated. Note further that $1 / c_{\theta}$ is the probability that this inequality be satisfied, so that $c_{\theta}$ represents the average number of pairs of samples $\left(Z_{\theta}, \bar{U}\right)$ that must be generated per value of $G_{i i}$ to be simulated. Accordingly, it is desired to have a value of $c_{\theta}$ as close as possible to 1 .

The Student's $t$ distribution with 3 degrees-offreedom was selected for the random variable $Z$ first because it can easily be simulated as

$$
Z=\sqrt{2 / U(1-U)}(U-1 / 2)
$$

where $U$ is a random number uniformly distributed in $[0,1]$. Another advantage of the Student's $t$ distribution is that it led to values of $c_{\theta}$ that remained reasonably small, i.e., to efficient simulation algorithms, over a broad range of values of the parameters $p(i)$ and $\tau_{i} / \mu_{i i}^{2}$.
The approach selected here for the determination of the parameters $c_{\theta}, \theta$, and $y_{0}$ is to force the two distributions $p G_{i i}\left(g_{i i}\right)$ and $p Z_{\theta}\left(g_{i i}\right)$ to have their respective modes at the same value $\left(g_{i i}^{*}\right)=y_{0}$ and to have $p G_{i i}\left(g_{i i}^{*}\right)=c_{\theta} p Z_{\theta}\left(g_{i i}^{*}\right)$. These 2 conditions will provide the values of $c_{\theta}$ and $y_{0}$ in terms of $\theta$. Specifically, it is found that

$y_{0}=\frac{1}{4 \tau_{i}}\left(\sqrt{\mu_{i i}^{2}+8 q \tau_{i}}-\mu_{i i}\right) \geq 0$

where $q=(n+2 \lambda-3) / 2$.

and

$c_{\theta}=2 \sqrt{2} \theta \overline{C_{i}} y_{0}^{q} \exp \left(-\mu_{i i} y_{0}-\tau_{i} y_{0}^{2}\right)$.

An acceptable simulation algorithm is obtained for all values of $\theta$ for which the inequality of Eq. (45) is satisfied. However, the most efficient of these algorithms is the one that minimizes the corresponding $c_{\theta}$, see Eq. (51). The exact solution of this optimization problem is difficult to achieve but two good possible values of $\theta$ are

$\theta=\sqrt{\frac{9+12 y_{0}\left(\mu_{i i}+4 \tau_{i} y_{0}\right)}{8\left(\mu_{i i}+4 \tau_{i} y_{0}\right)^{2}}}$.

$\theta=\sqrt{3\left(y_{0}+w^{*}\right) / 2\left(\mu_{i i}+4 \tau_{i} y_{0}+2 \tau_{i} w^{*}\right)-w^{* 2} / 2}$

where $w^{*}=\frac{(D-a)}{2 \tau_{i}}, \quad D=\frac{\gamma}{6}+\frac{2 a^{2}}{3 \gamma}+\frac{a}{3}$

$\gamma^{3}=108 b+8 a^{3}+12 \sqrt{81 b^{2}+12 b a^{3}}$

and $a=\mu_{i i}+4 \tau_{i} y_{0} \quad b=3 \tau_{i}\left(\mu_{i i}+2 \tau_{i} y_{0}\right)$.

A comparison of the simulation algorithms based on Eqs. (52) and (53)-(57) has shown that the algorithm based on Eqs. (49), (51), (53)-(57) performs very well, typically requiring an average of 1.4 pairs of independent uniform random numbers per sample $G_{i i}$ to be simulated. In unusual cases, the number of pairs of uniform variates may rise to 3 . The algorithm based on Eq. (52) in place of Eqs. (53)-(57) performs almost as well in the cases of greater practical interest but may lead to a large simulation effort when $q$ is small (e.g., equal 0 ) and $\left|\mu_{i i}\right| / \tau_{i}$ is typically less than 3.

The simulation of random values $G_{i i}$ according to the probability density function of Eq. (38) then proceeds as follows. For given values of $\mu_{i i}$ and $\tau_{i}$, the mode $y_{0}$ is first determined according to Eq. (49), and $\theta$ and $c_{\theta} / \bar{C}_{i}$ are obtained in order from Eqs. (52), or (53)-(57), and (51). After this preparation 
phase, pairs of independent random numbers $(U, \vec{U})$ uniform in $[0,1]$ are simulated. From $U$, a variate $Z$ of the Student's $t$ distribution is obtained from Eq. (48) and the corresponding variable $Z_{\theta}$ is determined as $Z_{\theta}=y_{0}+\theta Z$. It is next necessary to assess if the inequality of Eq. (47) is satisfied. To this end, the term $\left(c_{\theta} / \bar{C}_{i}\right) P Z_{\theta}\left(Z_{\theta}\right) \bar{U}$ is evaluated from the above values of $c_{\theta} / \bar{C}_{i}$ and $\bar{U}$ and with $p z_{\theta}\left(z_{\theta}\right)$ denoting the value of $p\left(Z_{\theta}\right)\left(g_{i i}\right)$, Eq. (46), for $g_{i i}=Z_{\theta}$. The ratio $p_{G i i}\left(Z_{\theta}\right) / \bar{C}_{i}$ is similarly obtained from Eq. (38). If

$\left(c_{\theta} / \bar{C}_{i}\right) p_{Z_{\theta}}\left(Z_{\theta}\right) \bar{U} \leq p_{G_{i i}}\left(Z_{\theta}\right) / \bar{C}_{i}$,

a value of $G_{i i}$ is obtained as $G_{i i}=Z_{\theta}$. Otherwise, the value of $Z_{\theta}$ is rejected and no corresponding sample of $G_{i i}$ is generated. Either way, the process is repeated starting with the simulation of a new pair of uniform random variables $(U, \bar{U})$ until the appropriate number of samples of $G_{i i}$ has been obtained. Note that Eq. (58) is equivalent to the inequality of Eq. (47) but is preferable to it because it does not require the numerical evaluation of the normalization constant $\bar{C}_{i}$.

\section{IDENTIFICATION OF NONPARAMETRIC MODEL PARAMETERS}

\subsection{Identification of the parameters $\lambda, \mu_{i i}$, and $\tau_{i}$}

The above derivations have been carried out in terms of the parameters $\lambda, \mu_{i i}$, and $\tau_{i}$, and but these coefficients are not part of the original problem statement and thus they should, in principle, be evaluated in terms of the stated constraints, Eqs. (4), (29), and (30). Note in this regard that the constraint of Eq. (4) has two aspects: the finiteness of $\nu$ and its specific value. The finiteness of $\nu$ guarantees the existence of the mean squared Frobenius norm of the inverse matrix $\underline{\underline{A}}^{-1}$ while the specific value of this coefficient providing an overall measure of the randomness of the matrices $\underline{\underline{A}}$.

Note however that the available information about the randomness of the model lies in the standard deviations of some of its natural frequencies and thus it would be desirable to identify all model parameters from that information alone. Such an approach has already been proposed (Capiez-Lernout et al., 2005): the standard deviation of the first natural frequency was used to evaluate the parameter $\lambda$. of an unconstrained model. A similar procedure is accordingly proposed here. Specifically, it will be assumed that the standard deviations of $m+1$ natural frequencies are known. Then, one of these standard deviation conditions will be used to yield the value of $\lambda$ while the $m$ others will be enforced through constraints of the form of Eq. (10).
To complete this strategy, it remains to determine which standard deviation should be singled out and used to compute $\lambda$. In this regard, it is valuable to analyze the behavior of the distribution of Eq. (38) as $q \rightarrow \infty$ large values of this parameter occur for small to moderate system variability. In this limit, it is found that a positive value of $\tau_{i}$ is obtained only if

$s_{i}^{2} \leq 1+1 / q$.

In this regard, note that $1+1 / q$ is the value of $E\left[G_{i i}^{2}\right]$ obtained from the unconstrained distribution of Eq. (24) in the same limit of $q \rightarrow \infty$. It is thus concluded that the specified value of $s_{i}^{2}$ must be less than what would be obtained by the unconstrained model with the same $\lambda$. This result is in fact not surprising since enforcing a higher value of $E\left[G_{i i}^{2}\right]$ would lead to an increase of entropy over the unconstrained, maximum entropy model.

This result also elucidates the determination of the parameter $\lambda$. Specifically, it will be evaluated from the standard deviation of the natural frequency which amongst the $m+1$ prescribed ones has the largest value of $s_{i}^{2}$. Practically, this condition is equivalent to selecting the natural frequency such that the ratio of its standard deviation to the corresponding natural frequency of the mean model is largest. Equality will be achieved in Eq. (59) for that value of $i$ and the corresponding parameter $\tau_{i}$ will be zero.

The above observation that $E\left|G^{2}{ }_{i i}\right|=1+1 / q$ for the unconstrained model as $q \rightarrow \infty$ implies that the ratios of the standard deviations of its random natural frequencies divided by the corresponding values for the mean model are the same, i.e., $1 / q$, for all natural frequencies. Thus, imposing an unconstrained model for the rows $m+1$ to $n$ of the matrix $\underline{H}$ implies that the corresponding natural frequencies will have the largest standard deviations possible. While this condition will lead to the largest value of the entropy, it would seem that the observed natural frequency of highest rank would provide the best basis for forecasting the variations of the unobserved, higher rank natural frequencies. Accordingly, it is proposed here that the parameters $\mu_{i i}$ and $\tau_{i}$ obtained for the row of $\underline{\underline{H}}$ associated with the observed natural frequency of highest rank be used also for the rows corresponding to unobserved, higher rank natural frequencies. Thus, the standard deviations of these random variables will be approximately (for finite value of $q$ ) constant as the rank increases.

\section{NUMERICAL RESULTS AND VALIDATION}

\subsection{Examples of application}

To exemplify the above developments, a $n=5$ degree of freedom dynamic system was considered exhibiting variations of its stiffness matrix. The 
above methodology was then applied with $\underline{A}$ and $\underline{\underline{B}}$ being the stiffness and mass matrices, respectively. Further, the analysis of the system was carried out in the modal coordinates of the mean model with mass normalized modes. Thus, $\underline{B}$ is the $5 \times 5$ unit matrix and $\bar{A}$ is the diagonal matrix containing the squares of the natural frequencies which were first assumed to be $1,3,5,7$, and $9 \mathrm{rad} / \mathrm{s}$ (mean model 1). Finally, the damping matrix was constructed by assuming a damping ratio of $1 \%$ on all modes.

Three sets of computations were carried out, two with the unconstrained nonparametric approach and one with the present constrained formulation. The first unconstrained model was obtained by enforcing a standard deviation of the first natural frequency equal to 0.058 , i.e., $5.8 \%$ of the corresponding value for the mean model. The second unconstrained model was similarly determined but with a lower standard deviation, i.e., 0.033. As stated earlier, it was observed that the ratios of the standard deviations of the natural frequencies of the random systems divided by their corresponding values for the mean models varied very little, from 0.00575 to 0.00584 for the first system and from 0.00331 to 0.00334 for the second one.

The present approach was applied next by imposing ratios of standard deviations to mean model value of $5.8 \%$ and $3.3 \%$ for the first and second natural frequency, respectively. Since the first natural frequency exhibits the largest relative variations, the first row of $\underline{H}$, i.e., the one most closely associated with the first frequency, was characterized by an unconstrained model, i.e., $\tau_{i}=0$. Given the lack of information on the third, fourth, and fifth natural frequencies, the parameters $\mu_{i i}$ and $\tau_{i}$ were assumed

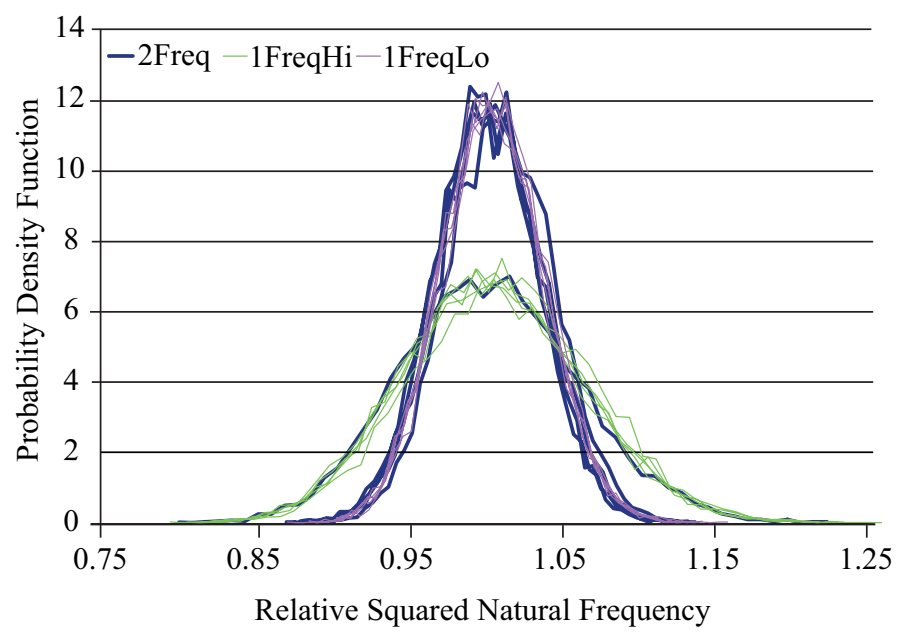

Figure 1. Probability density functions of the squared natural frequencies divided by their corresponding value for the mean model. Mean model 1. Constrained Model ("2Freq"), unconstrained models with higher ("1FreqHi”) and lower ("1FreqLo") level of variations. to be the same for $i \geq 2$. Accordingly, there were only 4 model parameters to be determined: $\mu_{11}, \lambda, \mu_{22}$ and $\tau_{2}$. They were iteratively obtained by imposing the prescribed standard deviations on natural frequencies (the exact constraints, not the approximate ones of Eq. (10)) and Eq. (29) for $i=1$ and 2. In fact, Eq. (29) for $i=1$ is readily satisfied by selecting $\mu_{11}$ and $\lambda$ to be related by Eq. (28) with $i=1$. Note further that the conditions of Eq. (29) for $i=3$, 4, and 5 are automatically satisfied when it is for $i=2$ as the model parameters $\mu_{i i}$ and $\tau_{i}$ and are the same for $i \geq 2$ A sample of 10,000 random matrices was assumed for all computations.

Shown in Fig. 1 are, for each of the three random systems, the distributions of the five natural frequencies divided by their corresponding value for the mean model. It is seen from this figure that the probability density function of the first natural frequency of the constrained system closely match the distributions of the natural frequencies (divided by their corresponding value for the mean model) of the unconstrained system with the largest, i.e.,

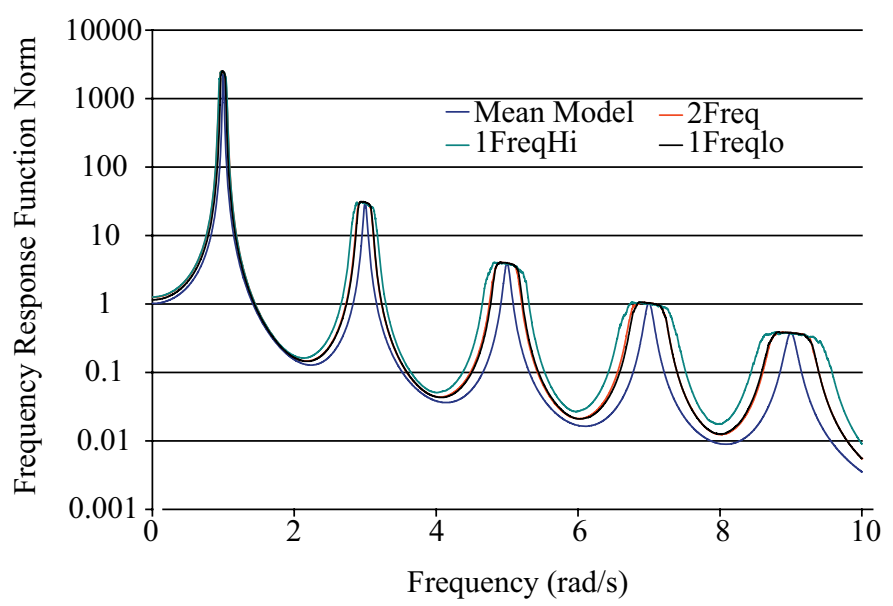

(a)

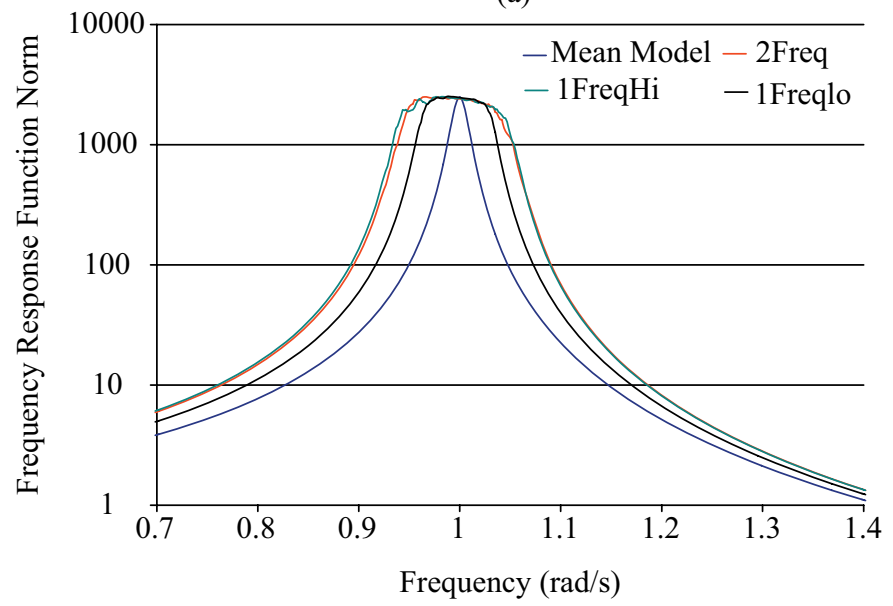

(b)

Figure 2. (a) Frobenius norm of the frequency response function matrix, (b) same, zoomed. Mean model 1. Constrained Model ("2Freq"), unconstrained models with higher ("1FreqHi”) and lower ("1FreqLo") level of variations. 
$5.8 \%$, level of variations. Similarly, the distributions of the $2^{\text {nd }}-5^{\text {th }}$ natural frequencies of the constrained system (divided by their corresponding value for the mean model) match the similar probability density functions of all 5 natural frequencies of the uncon-strained model with the lowest, i.e., $3.3 \%$, level of variations. It is thus concluded that the constrained model accurately matches the prescribed information on the variations of its natural frequencies.

A comparison of the frequency response functions of the 3 random systems was carried out next. Shown in Fig. 2 are the $95^{\text {th }}$ percentile of the Frobenius norm of the frequency response matrix $\left[\underline{\underline{K}}-\omega^{2} \underline{\underline{M}}+i \omega \underline{\underline{C}}\right]^{-1}$ for the constrained model, for the two corresponding unconstrained models, and for the mean model. It is clearly seen from this figure (and additional results not shown here for brevity) that the frequency response functions of the constrained model and of the unconstrained one corresponding to the smaller $(3.3 \%)$ variations in natural frequencies match very closely in the range $\omega \geq 1.5 \mathrm{rad} / \mathrm{s}$. For smaller frequencies however, e.g. see Fig. 2(b), the constrained model frequency response function closely match the unconstrained model with the largest variations natural in frequencies. These results are in fact in complete agreement with the distributions of natural frequencies presented in Fig. 1.

It was desired next to assess if the closeness of two natural frequencies of the mean model could affect the applicability of the approach and/or the validity of the above observations. To this end, the above computations were repeated exactly except for the second natural frequency of the mean model which was selected to be $1.06 \mathrm{rad} / \mathrm{s}$., i.e., very close to the first one (mean model 2). The parameters of all three random systems were recomputed and the distri-

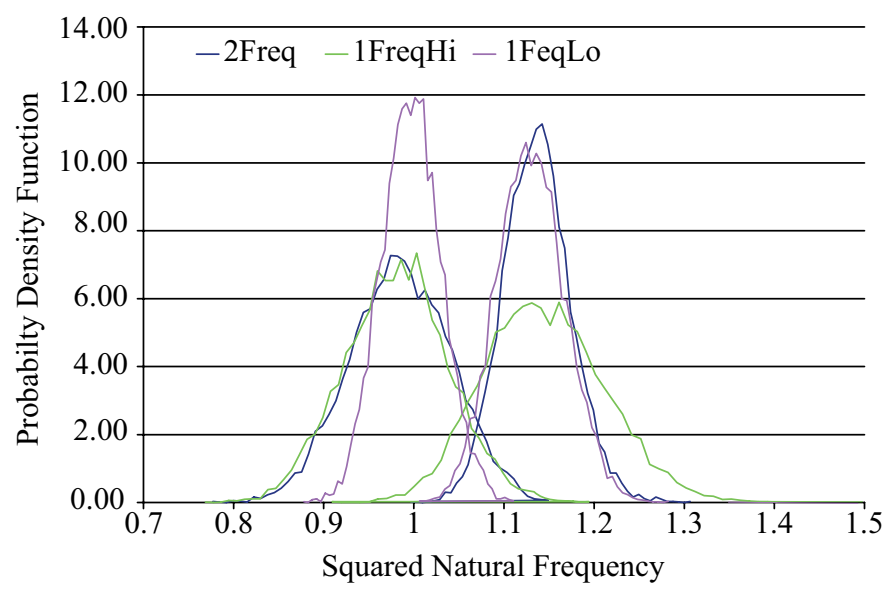

Figure 3. Probability density functions of the squared first and second natural frequencies. Mean model 2. Constrained Model ("2Freq"), unconstrained models with higher ("1FreqHi") and lower ("1FreqLo") level of variations.

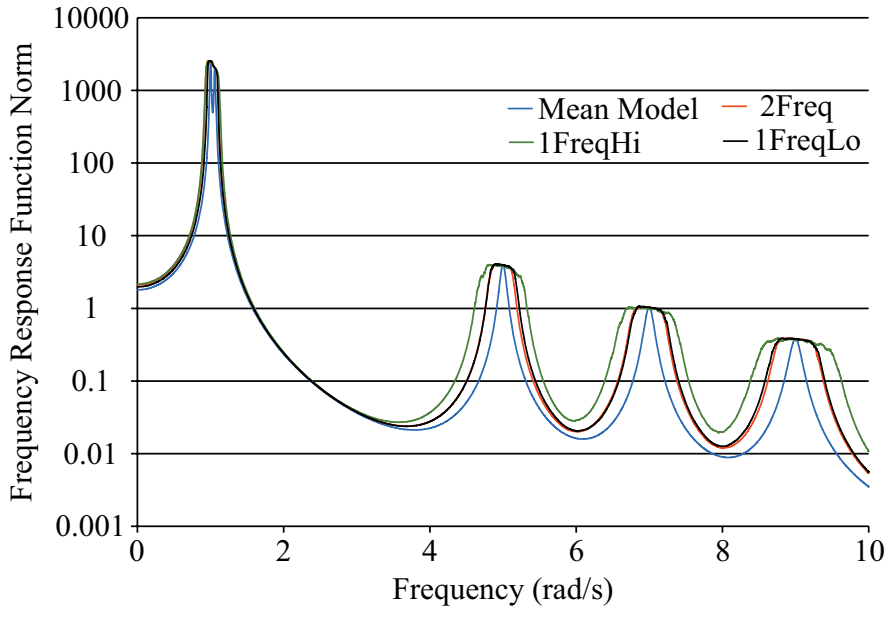

(a)

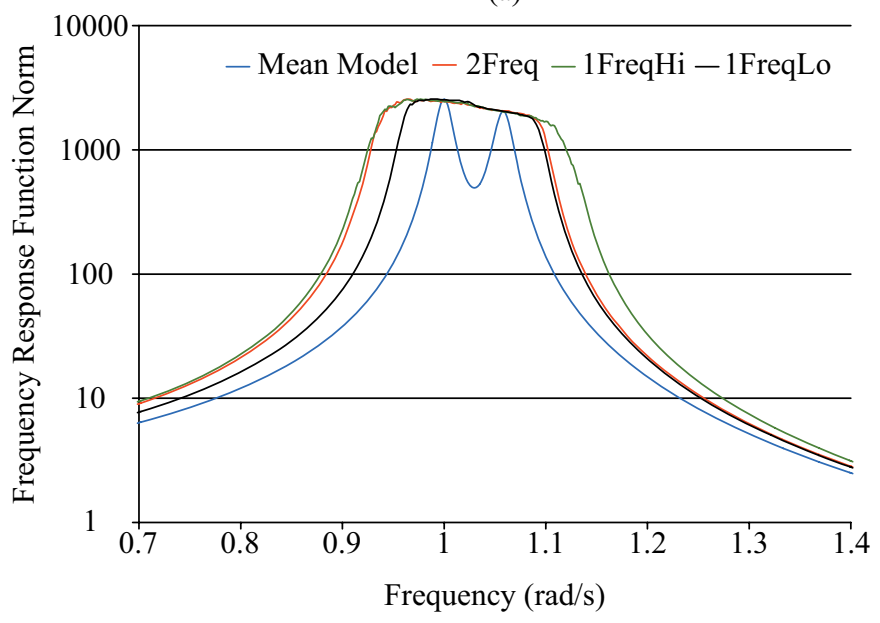

(b)

Figure 4. (a) Frobenius norm of the frequency response function matrix, (b) same, zoomed. Mean model 2. Constrained Model ("2Freq"), unconstrained models with higher ("1FreqHi") and lower ("1FreqLo") level of variations.

butions of natural frequencies and norm of frequency response functions were again determined. Not with standing the closeness of the natural frequencies of the mean system, the constrained model accurately matched the prescribed variations of the natural frequencies, see Fig. 3. Note again that the distribution of the first natural frequency matches the one from the unconstrained model with higher level of variations, while the probability density function of the second one matches its counterpart for the other unconstrained model, as seen in Fig. 1. Finally, the norm of the frequency response function matrix, see Fig. 4, exhibits the properties already described in connection with Fig. 2. In regards to the behavior near $1 \mathrm{~Hz}$, note first that the two peaks of the mean model have merged when considering any of its statistics (mean, $5^{\text {th }}$, and $95^{\text {th }}$ percentile) for either of the three random systems. Finally, note that the $95^{\text {th }}$ percentile of the norm of the frequency response function of the constrained model matches closely its unconstrained counterpart with the larger level 
of variations left of the peak (as dictated by the first natural frequency) and the one with the lower level of variations right of the peak (as dictated by the second natural frequency).

\section{SUMMARY}

The focus of this paper was on the derivation and validation of a maximum entropy based probabilistic model of positive definite matrices, such as the stiff-ness and mass matrices of a dynamic system. Following prior work in this area, the maximum of the entropy is sought under the additional constraints that the mean matrix is known, that each realization $\underline{\underline{A}}$ is positive definite, and that the mean squared Frobenius norm of its inverse exists. A new set of constraints was however added in the present effort, Eq. (10), to permit the enforcement of prescribed standard deviations of an arbitrary number of natural frequencies of the random system. Accordingly, the present methodology provides a full probabilistic model of random systems from basic variability information. Further, this model is independent of a rotation/change of coordinate system, as desired. It should be noted that if only the standard deviation of a single natural frequency is prescribed, the present model reduces to the one previously developed.

Central to the inclusion of natural frequency variations in the model are the Rayleigh quotient type constraints of Eq. (10) and the representations of the mean and random matrices $\underline{\underline{A}}$ and $\underline{\underline{A}}$ in the specific form of Eqs. (13), (14), and (16). These representations transfer the $m$ frequency constraints of Eq. (10) into prescribed second order moments of the first $m$ diagonal elements of the positive definite random matrix $\underline{G}$, the mean value of which is identity. Enforcing the positive definiteness of $\underline{G}$, is achieved by formulating directly the probabilistic model in terms of its Cholesky decomposition $\underline{H}$.

In fact, the joint probability density function of its elements is given by Eq. (22) in which it is recognized that each row is stochastically independent of the others. Further, the first $m$ rows, i.e., those associated with the natural frequency constraints, and the remaining ones are characterized by two different types of joint probability density functions. For rows $m+1$ to $n$, it is found, as in prior investigations, that all elements are independent of each other with the diagonal ones being distributed as square root of Gamma random variables. Further, the nondiagonal elements are found to follow a Gaussian distribution. The joint probability density function of the elements in the rows 1 to $m$ is more complex because of the frequency constraint which induces a statistical coupling between these random variables. Nevertheless, the joint probability density function of these elements was obtained, Eq. (26), and discussed extensively. Further, a simple approach was given to simulate these elements which hinges on the generation of samples of the diagonal elements $G_{i i}$ according to the distribution of Eq. (38). This task was achieved by an efficient, specially designed rejection algorithm, see Eqs. (45) and (52)-(57).

The details of the application of this model were discussed and exemplified on the stiffness matrix of two 5 degree of freedom models, one exhibiting widely spread frequencies while the other has two very close natural frequencies. In both cases, the model accurately matched the prescribed information on the natural frequency variations and thus provides a sound and complete probabilistic description of the random stiffness matrix of these stochastic systems.

\section{REFERENCES}

Capiez-Lernout, E., et al. 2005. Blade manufacturing tolerances definition for a mistuned industrial bladed disk, Journal of Engineering for Gas Turbines and Power, 127 (3): 621-628.

Devroye, L. 1986. Non-Uniform Random Variate Generation, Springer Verlag, New York.

Ghanem, R., and Spanos, P.D. 1991. Stochastic Finite elements: A spectral approach, Spring-Verlag, New York.

Kleiber, M., Tran, D.H., and Hien, T.D. 1992. The Stochastic Finite element Method, John Wiley and Sons, new York, 1992.

Rivas-Guerra, A.J., and Mignolet, M.P. 2004. Local/Global Effects of Mistuning on the Forced Response of Bladed Disks, Journal of Engineering for Gas Turbines and Power, 126(1): 131-141.

Schueller, G.I. (Ed.). 1997. A State-of-the-Art Report on Computational Stochastic Mechanics, Probabilistic Engineering Mechanics, 12(4): 197-321.

Soize, C. 2000. A nonparametric model of random uncertainties on reduced matrix model in structural dynamics, Probabilistic Engineering Mechanics, 15(3): 277-294.

Soize, C. 2001., Maximum entropy approach for modeling random uncertainties in transient elastodynamics, Journal of the Acoustical Society of America, 109(5): 1979-1996.

Soize, C. 2005. Probabilistic models for computational stochastic mechanics and applications, Proceedings of the $9^{\text {th }}$ International Conference on Structural Safety and Reliability ICOSSAR'05, Rome, Italy, 23-42. Millpress, Rotterdam. 